\title{
Polarization response of RHIC electron lens lattices
}

\author{
V. H. Ranjbar* and F. Méot ${ }^{\dagger}$ \\ Brookhaven National Lab, Upton, New York 11973, USA \\ M. Bai ${ }^{\ddagger}$ \\ Forschungszentrum Juelich, Juelich 52428, Germany \\ D. T. Abell ${ }^{\S}$ and D. Meiser ${ }^{\text {I }}$ \\ Tech-X Corporation, Boulder, Colorado 80303, USA
}

(Received 22 October 2015; revised manuscript received 8 August 2016; published 10 October 2016)

Depolarization response for a system of two orthogonal snakes at irrational tunes is studied in depth using lattice independent spin integration. In particular we consider the effect of overlapping spin resonances in this system, to understand the impact of phase, tune, relative location and threshold strengths of the spin resonances. These results are benchmarked and compared to two dimensional direct tracking results for the RHIC e-lens lattice and the standard lattice. Finally we consider the effect of longitudinal motion via chromatic scans using direct six dimensional lattice tracking.

DOI: 10.1103/PhysRevAccelBeams.19.101002

\section{INTRODUCTION}

A major limitation for the luminosity in colliders is due to head-on beam-beam collisions that lead to emittance blow-up at sufficiently high intensity. In order to compensate for the beam-beam collision, an electron lens (e-lens) [1-3] has been installed in each ring of the Relativistic Heavy Ion Collider (RHIC). To work effectively, however, this compensation requires (among other conditions) that the betatron phase advance be an integer multiple of $180^{\circ}$ ingoing from an interaction point (IP) to the e-lens. New accelerator optics for RHIC, the so-called e-lens lattices, were developed to provide this needed phase advance for both the Blue and Yellow rings.

During the $2013255 \mathrm{GeV}$ polarized proton run, the new e-lens lattices for both rings were commissioned. The e-lens lattice required different integer tunes for both rings. For the Blue ring the integer tunes were changed from $\left(Q_{x}, Q_{y}\right)=(28,29)$ to $(27,29)$, and for the Yellow ring, from $(28,29)$ to $(29,30)$. Compared with the standard lattice used during run 2012, both the Blue and Yellow ring e-lens lattices had lower values for the three very strong intrinsic spin resonances above $100 \mathrm{GeV}$ (see Table I). However, the

\footnotetext{
*Corresponding author.

vranjbar@bnl.gov

fmeot@bnl.gov

\$m.bai@fz-juelich.de

\$dabell@txcorp.com

`dmeiser79@gmail.com

Published by the American Physical Society under the terms of the Creative Commons Attribution 3.0 License. Further distribution of this work must maintain attribution to the author(s) and the published article's title, journal citation, and DOI.
}

neighboring weak resonances were larger than in the run 2012 lattice (see Fig. 1). This we attribute, at least in part, to the now asymmetric phase advances around the RHIC rings imposed by the operational requirements of the new e-lens lattices.

This was the first time the RHIC lattice had run with significantly altered intrinsic spin resonance structure [4]. The initial expectation was that polarization transmission through the RHIC ramp would improve, since each individual intrinsic resonance was well below the depolarization threshold in the presence of two snakes. This threshold has been described in previous theoretical studies [5-8]; but those studies do not address the potential impact of overlapping intrinsic resonances.

The results from the 2013 polarized proton run did not, in fact, show strong evidence of the expected improvement; indeed, polarization transmission through the RHIC ramp may have suffered. To understand this effect, we used the newly-developed code TEASPINK [9] to perform twodimensional (2D) and six-dimensional (6D) spin-orbit tracking on a platform using both message passing interface (MPI) and graphical processing units (gpu). Because of its

TABLE I. Differences in resonance strength magnitude $|w|$ between the e-lens and standard lattices for the three strongest intrinsic spin resonances.

\begin{tabular}{lcc}
\hline \hline & \multicolumn{2}{c}{$\Delta($ resonance strength $)=\left|w_{\text {new }}\right|-\left|w_{\text {old }}\right|$} \\
\cline { 2 - 3 } Resonances $[G \gamma]$ & Blue ring & Yellow ring \\
\hline $231+\nu_{y}$ & -0.0387 & -0.0415 \\
$411-\nu_{y}$ & -0.06134 & -0.0655 \\
$393+\nu_{y}$ & -0.05347 & -0.0347 \\
\hline \hline
\end{tabular}




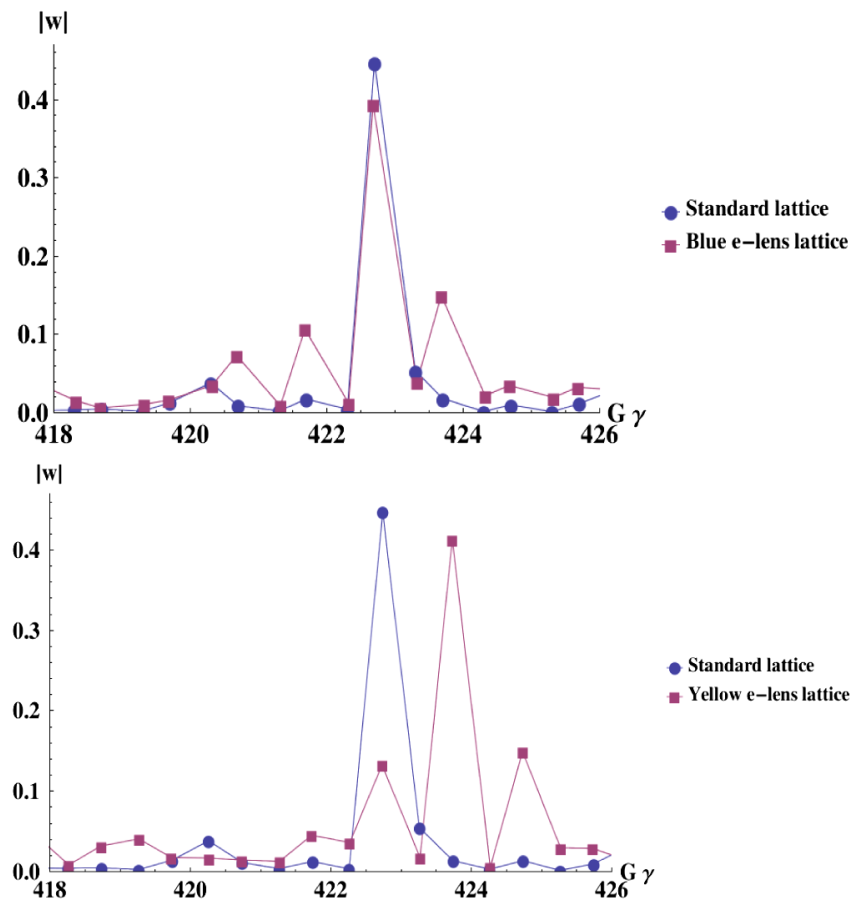

FIG. 1. Comparison of the Blue (top) and Yellow (bottom) e-lens intrinsic resonance strengths with standard RHIC lattice for $393+\nu_{y}$.

gpu capabilities, this code can track both orbit and spin for tens of thousands of particles at unprecedented speeds. For this paper, we tracked 50-32,000 particles across the strongest intrinsic resonances on the RHIC ramp. In addition, we used another newly-developed code to perform unitary integration across multiple resonances in a latticeindependent manner. The results of these studies motivated us to revisit the theory and carefully analyze the drivers of depolarization for this system. In particular, we studied the crossing of one or more intrinsic resonances in the presence of two snakes whose axis of rotation is orthogonal to each other (known as orthogonal snakes). While there has been much effort in past years devoted to understanding polarization behavior in the presence of snakes, several questions remain either unanswered or unclear in the current literature.

For example, our reading of the literature indicates that there is a hard limit that gives a machine's maximum tolerable resonance strength in terms of the number $N_{s}$ of snakes: $\left|w_{\max }\right| \leq N_{s} / 2$. This relationship has been derived in a number of ways. It was first deduced from direct tracking by Lee and Courant [5] and later supported by analytical calculations of the so-called "perturbed spin tune." However, that work was done before there was a clear understanding of the importance of the invariant spin field (ISF) and, especially, of the role the ISF plays in properly setting up and interpreting spin dynamical tracking studies. (In this latter context, we remind the reader that measuring polarization loss in a tracking study requires that one prepare the initial spins parallel to the local ISF, and then compare the final spins also against the local ISF.) Later analytical and numerical work done by Mane [10] showed that the stable spin direction becomes numerically unstable as the resonance strength approaches 1 for a two-snake system. Outside of the narrow integer region of resonance space, the mechanism for polarization loss was unclear since we were to understand that the fact that our amplitude dependent spin tune [10] would remain fixed at $1 / 2$. If this was true then what was the mechanism for polarization loss? Indeed other authors pointed to discontinuities in the spin tune as signatures for polarization loss $[11,12]$.

A secondary question following from the first is how does this aperture respond as secondary resonances are introduced. What are the thresholds in strength and distance from the main resonance? What role does phase play? In this paper we answer some of these questions by employing our lattice independent spin integration code. First we revisited the Single Resonance Model (SRM) with snakes. Next we explored the effect of adding a secondary resonance. We found strong evidence to support the conjecture that provided we begin tracking on the stable spin direction and compare final spin orientation with the final stable spin direction that there is no mechanism to move the spin vector away from the stable spin direction (outside of the narrow band around the integer). A weaker statement is that, at least for the orthogonal system of snakes with a single resonance at an irrational betatron tune, there is no polarization loss observable for any resonance strength which could fit into the RHIC aperture less than $50 \pi \mathrm{mm}$ mrad the emittance necessary for the strongest resonance crossing in the $250 \mathrm{GeV}$ RHIC ramp to equal $|w|=1$.

Next we observed that for the strong resonances crossings in the RHIC lattice, the threshold at which a secondary resonance can impact the polarization aperture is at $\approx 0.01$. Finally we present a possible approach to greatly expand the polarization aperture by minimizing the neighboring resonances.

\section{BENCHMARKING LATTICE INDEPENDENT INTEGRATION}

We have developed a code to integrate the 2D spinor form of the Thomas-BMT (Bargmann, Michel, and Telegdi) equation [13]:

$$
\frac{d \Psi}{d \theta}=-\frac{i}{2}\left(\begin{array}{ll}
f_{3} & -\xi \\
\xi^{*} & -f_{3}
\end{array}\right) \Psi
$$

Here $\xi(\theta)=F_{1}-i F_{2}$ and $f_{3}=\left(1+F_{3}\right)$, with

$$
\begin{aligned}
& F_{1}=-\rho z^{\prime \prime}(1+G \gamma), \\
& F_{2}=(1+G \gamma) z^{\prime}-\rho(1+G)\left(\frac{z}{\rho}\right)^{\prime}, \\
& F_{3}=-(1+G \gamma)+(1+G \gamma) \rho x^{\prime \prime} .
\end{aligned}
$$


Further we neglect the $(1+G \gamma) \rho x^{\prime \prime}$ term in $F_{3}$ since it is usually much smaller than the $(1+G \gamma)$ term.

Although the spinor wave function $\Psi$ is similar in form to the quantum mechanical state function, in this case $\vec{S}$ is a classical vector. As in the quantum mechanical case, however, this two component spinor is defined as

$$
\Psi=\left(\begin{array}{l}
u \\
d
\end{array}\right)
$$

Here $u$ and $d$ are complex numbers representing the up and down components. The components of the spin vector behave in the usual way with

$$
\begin{aligned}
& S_{1}=\Psi^{*} \sigma_{1} \Psi=u^{*} d+u d^{*} \\
& S_{2}=\Psi^{*} \sigma_{2} \Psi=-i\left(u^{*} d-u d^{*}\right) \\
& S_{3}=\Psi^{*} \sigma_{3} \Psi=|u|^{2}-|d|^{2} .
\end{aligned}
$$

Because $H=(\vec{\sigma} \cdot \vec{n})$ is Hermitian,

$$
|\vec{S}|=|u|^{2}+|d|^{2}=\Psi^{\dagger} \Psi
$$

and the magnitude of the spin vector remains constant. The normalization condition for the spinor function is chosen to be $\Psi^{\dagger} \Psi=1$.

Using a 4th order Magnus Gaussian quadrature integrator described in [14] we can integrate Eq. (1) for an arbitrary $\xi(\theta)$. In this code the effect of snakes and rotators are handled separately and are added into the lattice as thin spin kicks.

We first consider the polarization response for a single isolated resonance. This has been much studied in the past-especially the nonaccelerating case, where an analytical expression and approximations have been developed

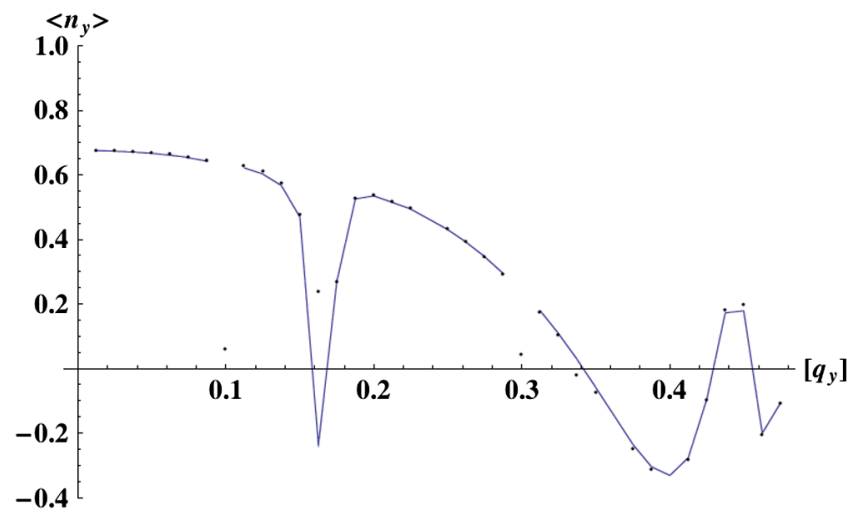

FIG. 2. Plot of stroboscopic average vertical component $\left\langle n_{y}\right\rangle$ versus the fractional orbital tune $\left[q_{y}\right]$ with resonance amplitude of 0.4. Dots represent numerically calculated values using 128 particle average and lines are the analytically calculated values using S. Mane's formula [10]. by S. Mane [10]. We revisit this as a cross check for our new integrator (see Fig. 2) and find good agreement.

We next compare the accelerating case to see if we can reproduce results similar to direct tracking while crossing the $393+\nu_{y}$ resonance for the standard lattice.

In Fig. 3 we show spin aperture plots comparing 2D lattice independent integration against direct 2D tracking. For these and all future spin aperture plots (unless otherwise specified), the following apply. The acceleration rate is held fixed at $\frac{\mathrm{d} \gamma}{\mathrm{d} t}=1.24 / \mathrm{sec}$. On the horizontal axis, the vertical emittance is rescaled to the absolute resonance strength $|w|$, based on the strength of the main resonance at $10 \pi \mathrm{mm} \mathrm{mrad}$ as calculated by DEPOL [13], a program for calculating intrinsic and imperfection spin resonances. For the lattice independent traces, all secondary resonances are scaled along with the main resonance $|w|$. On the vertical axis is plotted the difference between the average final vertical spin component of a sample of 128 trajectories distributed uniformly over an initial betatron phase and the final stable spin direction $\left(\left|\left\langle n_{y}\right\rangle-\left\langle S_{y}\right\rangle\right|\right)$. For the largest amplitudes considered, we found that 128 trajectories sufficed to converge to the average polarization obtained using larger sample sizes (256, 500, and 1000). These
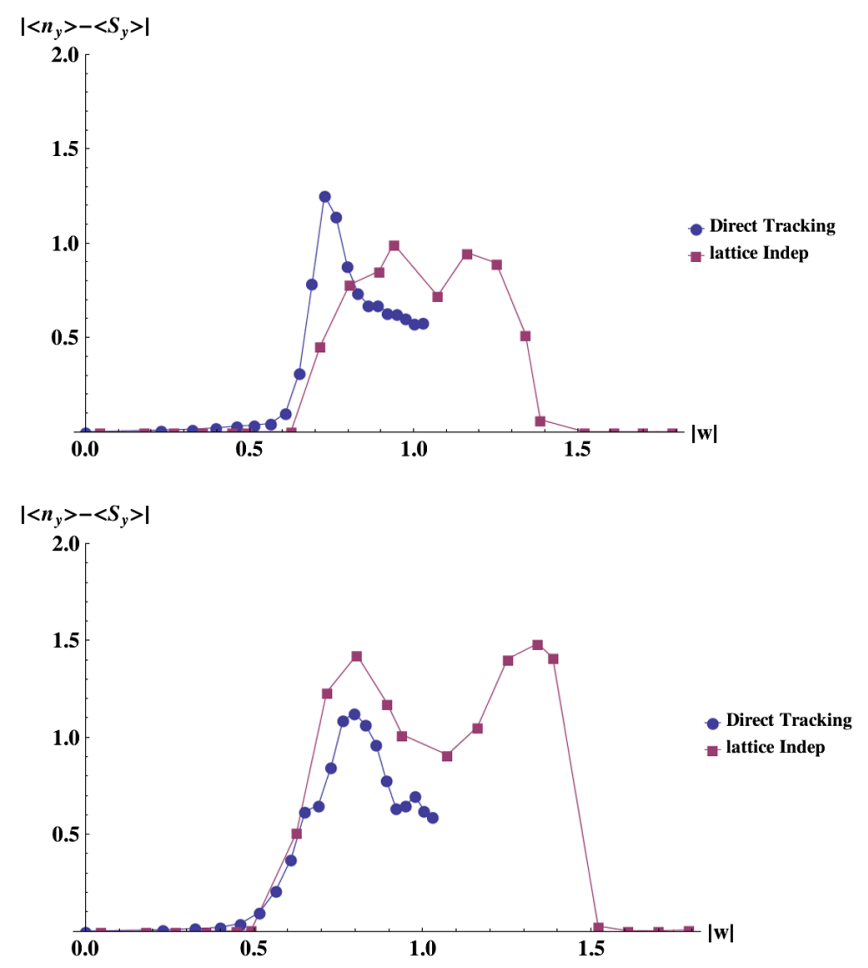

FIG. 3. Comparisons of 2D lattice independent integration versus direct tracking for simulations of the Standard lattice crossing the strong $393+\nu_{y}$ resonance. Here the lattice independent code used five resonances within $\pm 1 G \gamma$ of the strongest resonance. In the case of direct tracking, we set the horizontal and longitudinal emittances to zero. The two graphs show comparisons at $q_{y}=0.671$ (top) and $q_{y}=0.675$ (bottom). 

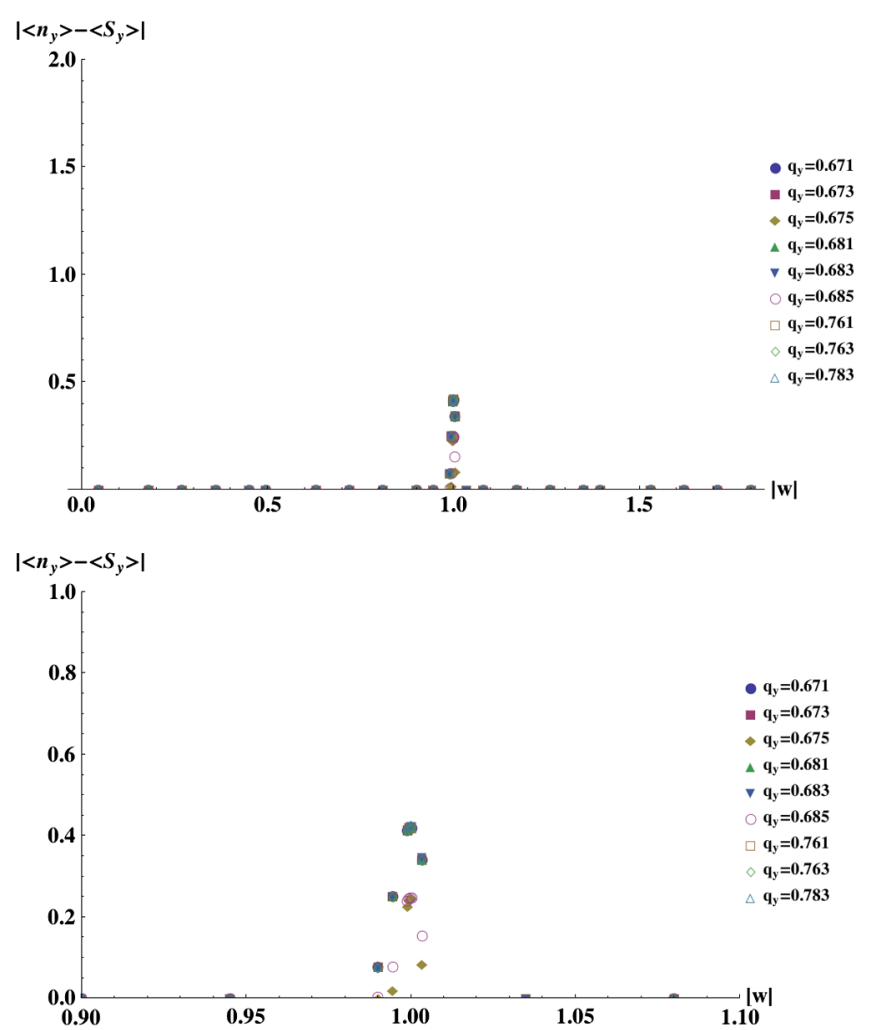

FIG. 4. Top: Plot of polarization response to a scan of resonance amplitude $|w|$ for an isolated resonance at a variety of fractional betatron tunes $\left(q_{y}\right)$. Bottom: A blow up of the top plot showing the width of the depolarization spike to be about $\leq 0.02$.

aperture plots provide an easy way to compare the spin preservation for different lattices.

The simple 2D lattice independent code, while differing in some fine details, reproduces the overall spin transmission aperture using five resonances within $\pm 1 G \gamma$ of the central resonance. We attribute the differences to two possible sources: (i) the initial or final stroboscopic averages were not completely converged, or (ii) the vertical precession due to the quadrupoles, since this model neglects the $(1+G \gamma) \rho x^{\prime \prime}$ term in the Thomas-BMT equation.

Finally, we revisited the SRM to explore the discontinuity at $|w|=1$, using the SRM with resonance strengths $0-5$ over the whole range of irrational tunes $0-1.0$. This showed that the region of polarization loss near the integer resonance is very narrow and that above the integer, polarization transmission is restored. An extract of this scan is shown in Fig. 4.

One could argue that for a realistic multiparticle system the effects of this discontinuity at $|w|=1$ would go unobserved and might be considered a numerical artifact.

\section{EFFECT OF OVERLAPPING RESONANCES}

Recent theoretical work [15] has shown that the T-BMT equation can be recast into a Hill's like equation with a

quasiperiodic kernel. For the case where there is a strong resonance perturbed by a weaker resonance, the perturbative effect can be understood as parametric resonances. This paper showed that the Foirsart-Stora formula for single strong resonance remains valid until the onset of the parametric resonance region. So our conjecture is that with snakes we should see the same behavior as the snake SRM until a parametric resonance is crossed. This should mean that primary cause of depolarization is due to the parametric resonances caused by the introduction of the secondary resonance. The consequence of this is that while the strength of the main resonance is generally important when other resonances are present, more important might be the threshold of where the overlapping resonance becomes significant. If we can drive down these nearby secondary resonances we should be able to open up the polarization aperture. From the perspective of lattice symmetry it would seem that with snakes one would want a highly symmetric lattice with large but isolated intrinsic resonances which is contrary to our previous assumptions and what one needs in lattices without full snakes.

\section{A. Two intrinsic resonances}

The introduction of a second intrinsic resonance dramatically changes the depolarization threshold and curve structure. We consider the response to the addition of one out of the four nearest resonances. There are two types of intrinsic resonance groupings, the first has an $N+\nu$ for the primary resonance (as for the strongest resonance $393+\nu$ ) and the second with $N-\nu$ as the primary resonances (as for the $411-\nu$ resonance). For the first case we define the primary resonances $K_{1}=1+q_{y}+n$ and the four secondary as: $1 . K_{0}=q_{y}+n, 2 . K_{2}=2+q_{y}+n, 3 . K_{3}=2-q_{y}+n$, 4. $K_{4}=3-q_{y}+n$. Here $q_{y}$ is the fractional part of the vertical tune and $n$ is an arbitrary integer setting the general location of the resonance group. From the point of view of relative distances between the primary resonance $\left(K_{1}\right)$ and the secondaries it yields the following differences: 1. $K_{1}-K_{0}=1$, 2. $K_{1}-K_{2}=-1,3 . K_{1}-K_{3}=2 q_{y}-1$, 4. $K_{1}-K_{4}=2\left(q_{y}-1\right)$. This is analogous to the $N-\nu$ resonance grouping where: $1 . K_{1}=1+n-q_{y}, 2 . K_{0}=$ $n-q_{y}, \quad 3 . \quad K_{2}=2+n-q_{y}, \quad 4 . \quad K_{3}=n+q_{y}, \quad 5 . \quad K_{4}=$ $n-1+q_{y}$, giving: $1 . K_{1}-K_{0}=1,2 . K_{1}-K_{2}=-1$, 3. $K_{1}-K_{3}=1-2 q_{y}, 4$. $K_{1}-K_{4}=2\left(1-q_{y}\right)$. This relative difference is identical to the $N+\nu$ grouping with the exception of a sign flip for the $K_{1}-K_{3}$ and $K_{1}-K_{4}$ differences.

Considering the $N+\nu$ grouping first, in Figs. 5-8 we can see the $K_{4}$ appears to have most impact across all phases and the effect of $K_{3}$ appears negligible across all phases. Here the phase difference is due to the complex phase difference between the two resonances (i.e., $\phi_{1}-\phi_{2}$, where the resonance strength is given as $\left.\left|w_{i}\right| e^{-i \phi_{i}}\right)$. Later 


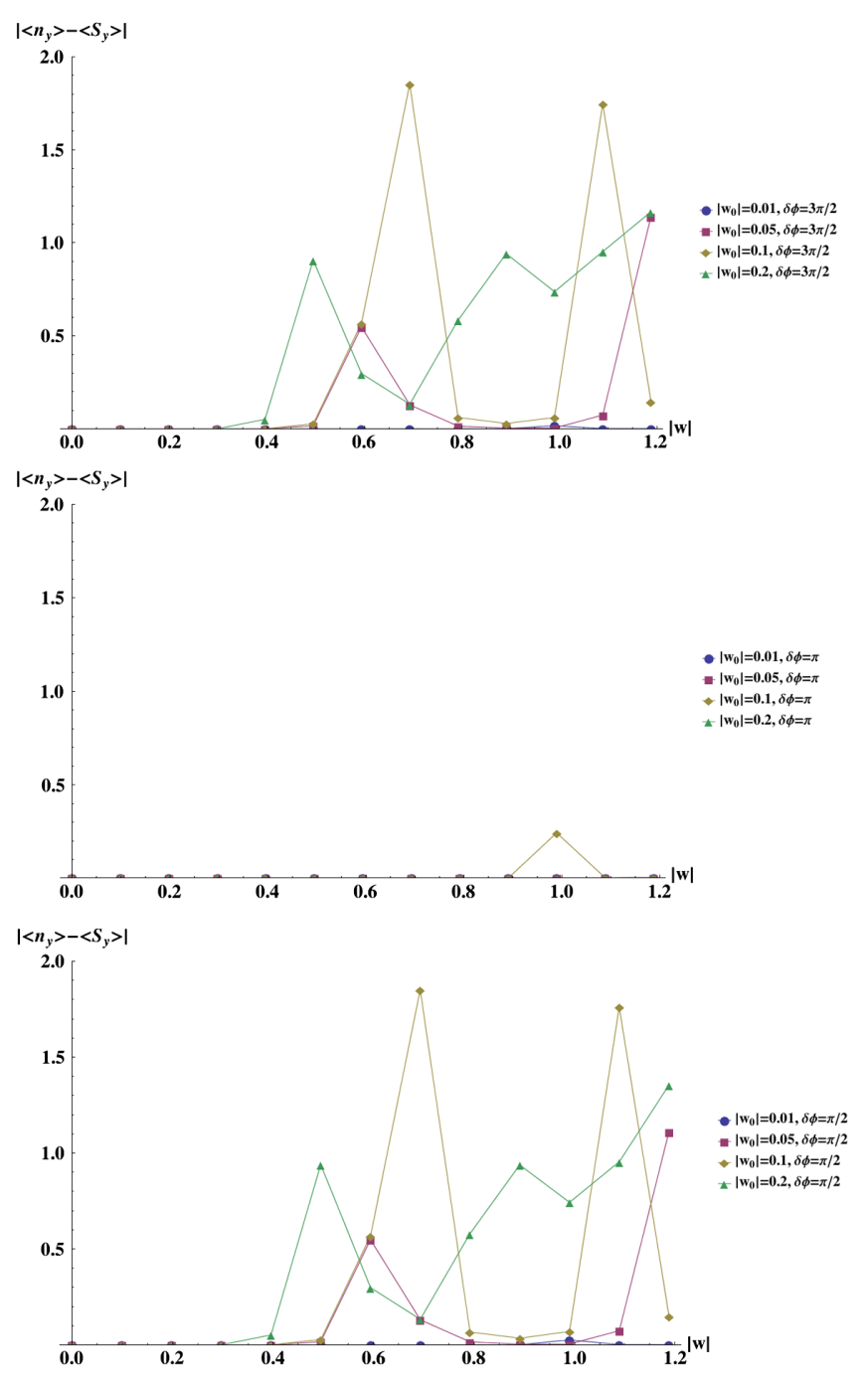

FIG. 5. Polarization aperture plots for $K_{1}$ with $K_{0}$ resonance. Here the fractional tune was $q_{y}=0.675$. We used absolute resonance strength of 0.45 for normalized $10 \pi \mathrm{mm} \mathrm{mrad}$ for $K_{1}$ and $K_{0}=(0.01,0.05,0.1,0.2)$. The X-axis $|w|$ represents the resonance strength of the primary resonance $\left(K_{1}\right)$ with the secondary $\left(K_{0}\right)$ scaled along with it. We considered three relative phase differences between the primary and secondary resonance $\Delta \phi=3 \pi / 2$ (top), $\pi$ (middle) and $\pi / 2$ (bottom).

we will revisit $K_{4}$ as we try to improve the polarization aperture for $393+\nu_{y}$ resonance crossing in the standard lattice. However $K_{0}$ and $K_{2}$ both one unit of $G \gamma$ away from the primary resonances $K_{1}$ share large swings in impact due to the relative phase.

As such we also considered a more careful study of the phase response in Fig. 9 where we can see polarization response to relative phase between the strong and weak neighboring intrinsic spin resonance. From this study we see again verified that $K_{3}$ 's effect maybe ignored while $K_{4}$ 's effect remains relatively high and constant. $K_{0}$ and $K_{2}$ also oscillate but with a much larger amplitude, so paying close attention to
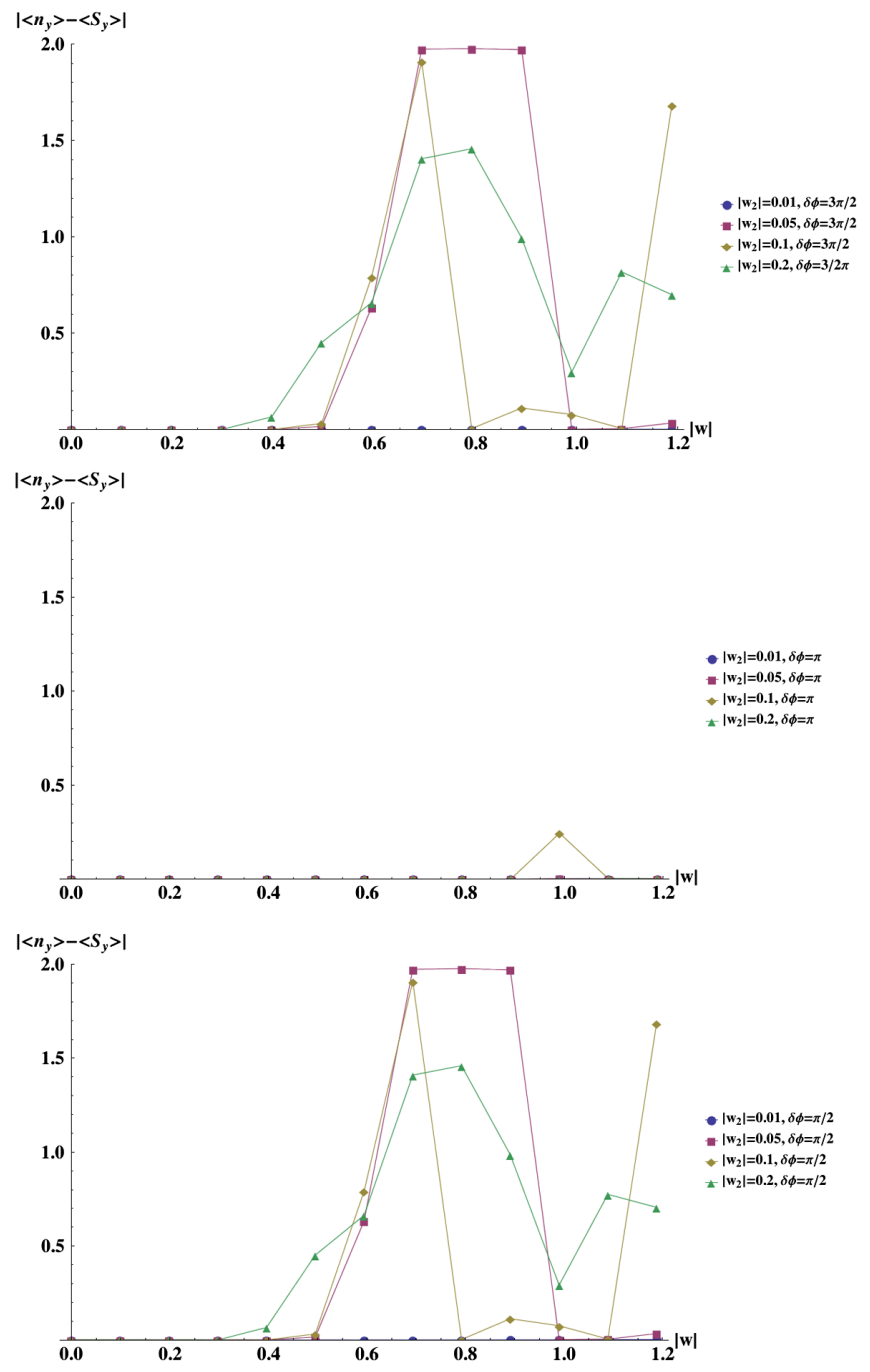

FIG. 6. Polarization aperture plots for $K_{1}$ with $K_{2}$ resonance. Here the fractional tune was $q_{y}=0.675$. We used absolute resonance strength of 0.45 for normalized $10 \pi \mathrm{mm}$ mrad for $K_{1}$ and $K_{2}=(0.01,0.05,0.1,0.2)$. The X-axis $|w|$ represents the resonance strength of the primary resonance $\left(K_{1}\right)$ with the secondary $\left(K_{2}\right)$ scaled along with it. We considered three relative phase differences between the primary and secondary resonance $\Delta \phi=3 \pi / 2$ (top), $\pi$ (middle) and $\pi / 2$ (bottom).

the relative phase should be important in minimizing their impact. We also explored the effect of small tune changes to the phase response for $K_{0}$ as can be seen in Fig. 10. Here we see the locations of the maximum and minimum appear unchanged but the width of the peaks are modified.

For the $N-\nu$ case we found the same style of response to relative location and phase. Apparently the change in sign does not impact the general rules for the response.

\section{B. Intrinsic with single imperfections}

The two nearby imperfection spin resonances whose overlap can lead to a reduction of polarization aperture, 


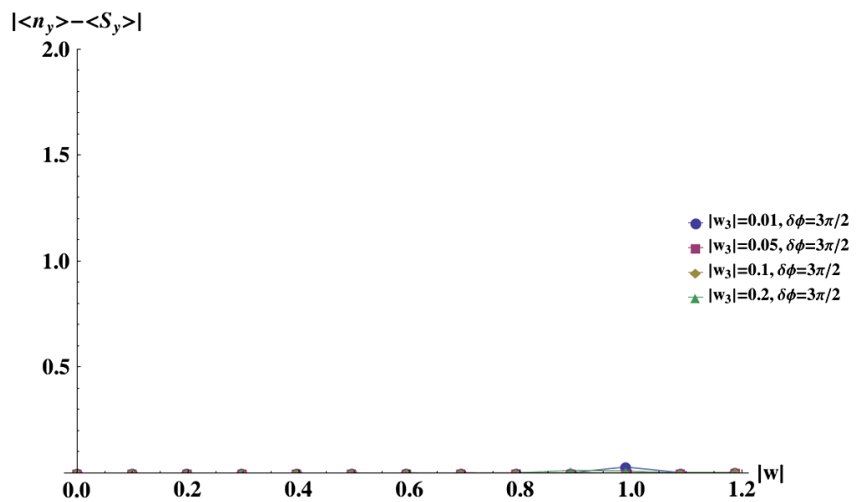

FIG. 7. Polarization aperture plots for $K_{1}$ with $K_{3}$ resonance. Here the tune was $\nu_{y}=0.675$. We used absolute resonance strength of 0.45 for normalized $10 \pi \mathrm{mm} \mathrm{mrad}$ for $K_{1}$ and $K_{3}=(0.01,0.05,0.1,0.2)$. The X-axis $|w|$ represents the resonance strength of the primary resonance $\left(K_{1}\right)$ with the secondary $\left(K_{3}\right)$ scaled along with it. We considered three relative phase differences between the primary and secondary resonance $\Delta \phi=3 \pi / 2 . \Delta \phi=\pi, \pi / 2$ (not shown) did not demonstrate any significant difference from this plot.

occur at two of the nearest integers which we call $K_{\mathrm{imp1}}=$ $1+n$ and $K_{\text {imp2 }}=-1+n$ (as before $n$ here sets the location of the resonances group). Their effect on polarization aperture can be seen in Fig. 11.

In Fig. 12 we now study the phase response for each of the imperfection resonances. Here we see that unlike the interfering intrinsic resonances, the response appears relatively insensitive to the phase. However as we saw before tune does matter.

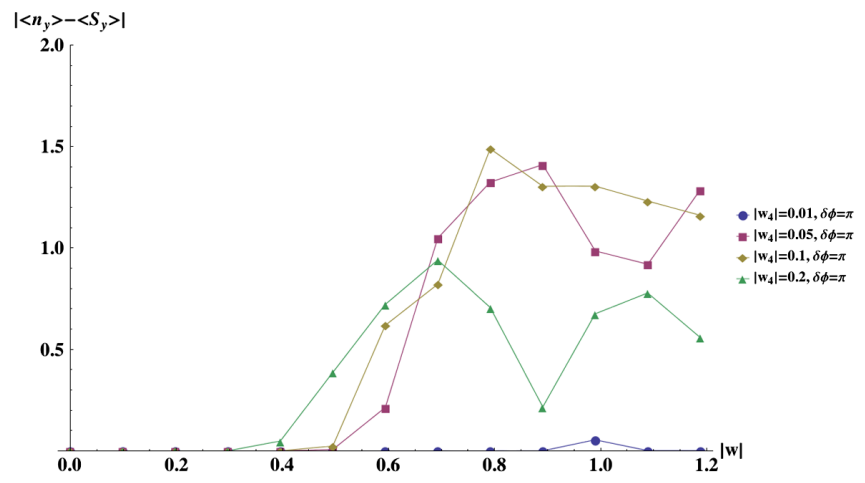

FIG. 8. Polarization aperture plots for $K_{1}$ with $K_{4}$ resonance. Here the tune was $\nu_{y}=0.675$. We used absolute resonance strength of 0.45 for normalized $10 \pi \mathrm{mmmrad}$ for $K_{1}$ and $K_{4}=(0.01,0.05,0.1,0.2)$. The $\mathrm{x}$-axis $|w|$ represents the resonance strength of the primary resonance $\left(K_{1}\right)$ with the secondary $\left(K_{4}\right)$ scaled along with it. We considered three relative phase differences between the primary and secondary resonance $\Delta \phi=\pi . \Delta \phi=3 \pi / 2, \pi / 2$ (not shown) did not demonstrate any significant difference from this plot.

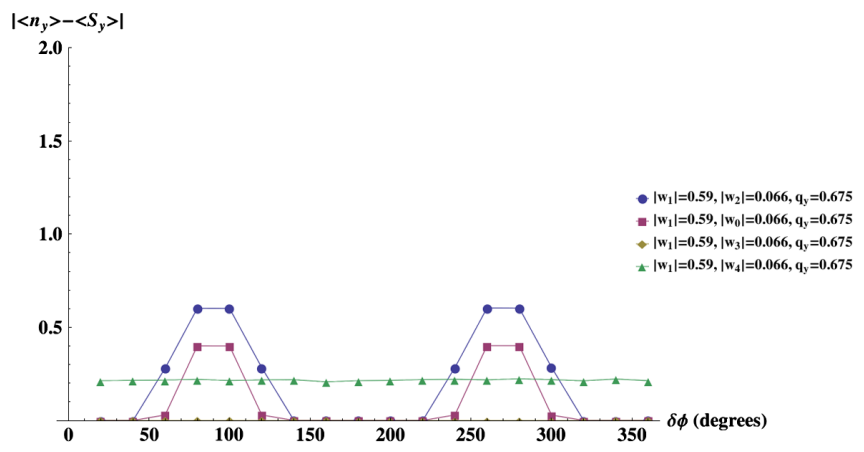

FIG. 9. Polarization response for particles whose primary resonance is $w=0.59$ and the secondary at $w=0.066$. This simulates the behavior of a particle at $17 \pi$ whose primary and secondary strengths at $10 \pi=0.45,0.05$ respectively. Here we consider the secondary resonance at $K_{0,2,3,4}$ with the relative phase between the primary and secondary resonance scanned between 0 and $2 \pi$. The acceleration rate is the nominal RHIC ramp rate of $\mathrm{d} \gamma / \mathrm{d} t=1.24 / \mathrm{sec}$, and the fractional vertical tune $q_{y}=0.675$.

Generally we see that the effect of both of the imperfections grow out from the location of $|w|=1$ with $K_{\text {imp1 }}$ having a stronger effect and with a similar threshold at $\approx 0.01$ (ignoring the singular loss at $|w|=1$ ).

\section{Intrinsic with coupled intrinsic resonance}

As we perform direct lattice tracking with more degrees of freedom we can try to simulate their behavior using the 2D lattice independent integration by including more Fourier components. For the case of horizontal motion, while we have neglected the $(1+G \gamma) \rho x^{\prime \prime}$ term for the lattice independent integration, one discovers that the primary effects of linear betatron coupling is due to the

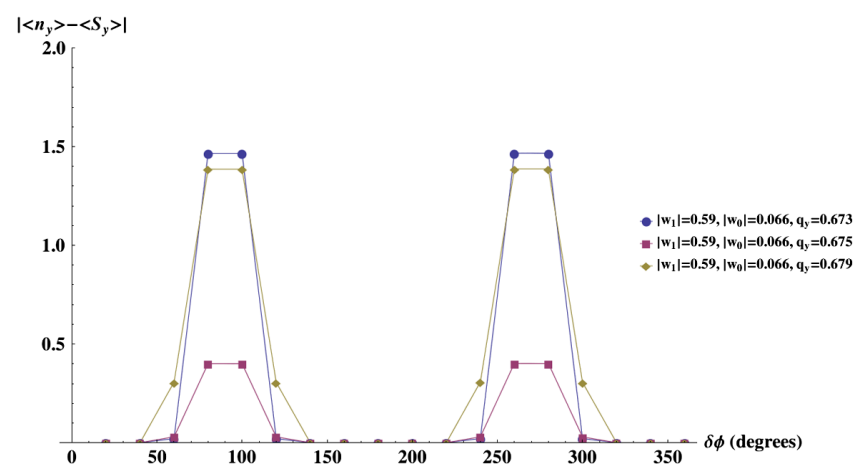

FIG. 10. Polarization response for particles whose primary resonance is $w=0.59$ and the secondary at $w=0.066$. This simulates the behavior of a particle at $17 \pi$ whose primary and secondary strengths at $10 \pi=0.45,0.05$ respectively. Here we consider the $K_{0}$ resonances with the relative phase between the primary and secondary resonances scanned between 0 and $2 \pi$ for fractional tunes $q_{y}=0.673,0.675$ and 0.679 . The acceleration rate is nominal RHIC ramp rate $d \gamma / d t=1.24$. 

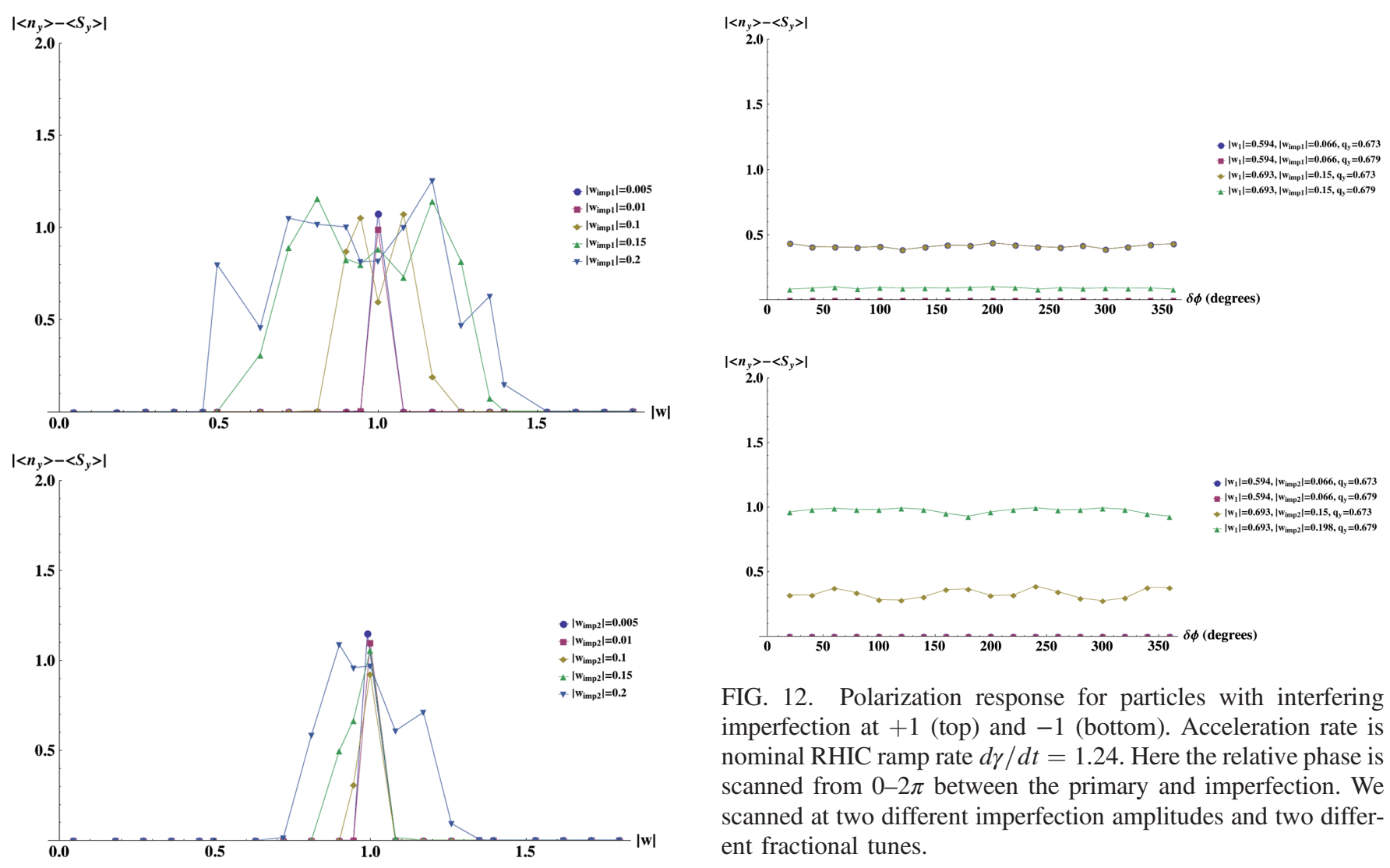

FIG. 12. Polarization response for particles with interfering imperfection at +1 (top) and -1 (bottom). Acceleration rate is nominal RHIC ramp rate $d \gamma / d t=1.24$. Here the relative phase is scanned from $0-2 \pi$ between the primary and imperfection. We scanned at two different imperfection amplitudes and two different fractional tunes.

FIG. 11. Top: Effect of $w_{\text {imp } 1}=0.005,0.01,0.1,0.15,0.2$ with $q_{y}=0.675333$ and phase $=180$ degrees. Bottom: Effect of $w_{\text {imp2 }}=0.005,0.01,0.1,0.15,0.2$ with $q_{y}=0.675333$ and phase $=0$ degrees.

projection of the horizontal tune onto the vertical motion. Thus the bulk of the effect of the addition of horizontal motion occurs via linear coupling. This effect can be contained in the $\xi(\theta)=F_{1}-i F_{2}$ term whose Fourier series can now include resonance amplitudes due to the horizontal motion. These resonance amplitudes can be computed using a modification to the DEPOL algorithm [16]. Thus we can include the effects of coupling by adding Fourier resonance terms which correspond to the horizontal betatron tune $N \pm \nu_{x}$.

Previous studies [17] have indicated coupled spin resonances can occur at $393+\nu_{x}$ at the level of 0.15 . In Fig. 13 we show the results of lattice independent integration including the effect of interfering coupled intrinsic spin resonances. We placed this resonance at $K_{1 x}=q_{x}+n$ relative to the primary at $K_{1}=1+q_{y}+n$ (reflecting the integer difference between the vertical and horizontal tune for RHIC). We discovered that the response appears insensitive to phase difference, as the results were fairly similar for phases $=\pi, \pi / 2$ and $3 \pi / 2$ (shown in Fig. 13). Additionally apart from the narrow spike at the integer resonance strength the effect of the resonances has the same threshold $\approx 0.01$ as the other interfering resonances studied so far.

\section{UNDERSTANDING IMPACT OF 6D MOTION}

Considering now the impact of longitudinal motion, we move finally to full 6D dynamics. Within the context of the lattice independent integration, it is possible to consider the

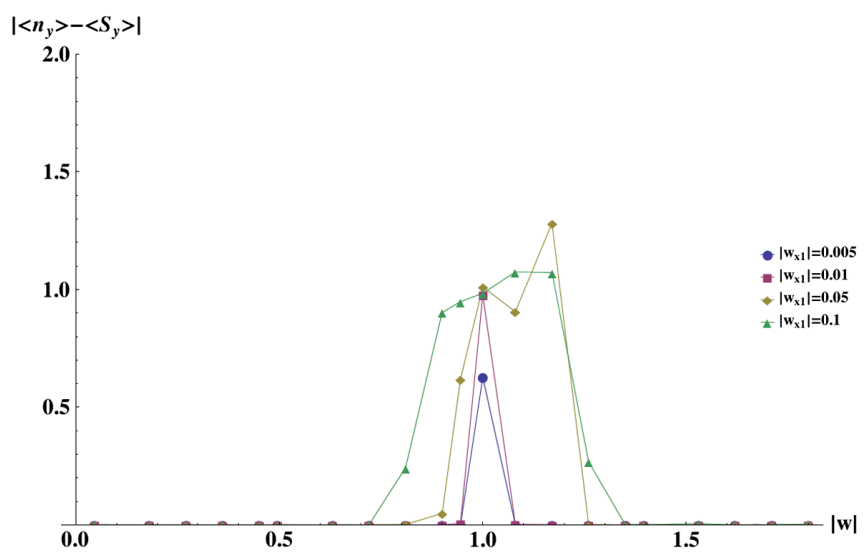

FIG. 13. Polarization aperture plots for $K_{1}$ with $K_{1 x}$ coupled spin resonance. Here the lattice independent code used 128 initial trajectories uniformly over the betatron phases at each resonance strength, the acceleration rate is $\frac{d \gamma}{d t}=1.24 / \mathrm{sec}$ and the fractional tune $q_{y}=0.675333$ and $q_{x}=0.685333$. We used absolute resonance strength of 0.45 for normalized $10 \pi \mathrm{mm} \mathrm{mrad}$ for $K_{1}$ and $K_{1 x}=(0.01,0.05,0.1,0.15,0.2)$. The X-axis $|w|$ represents the resonance strength of the primary resonance $\left(K_{1}\right)$ with the secondary $\left(K_{1 x}\right)$ scaled along with it. 

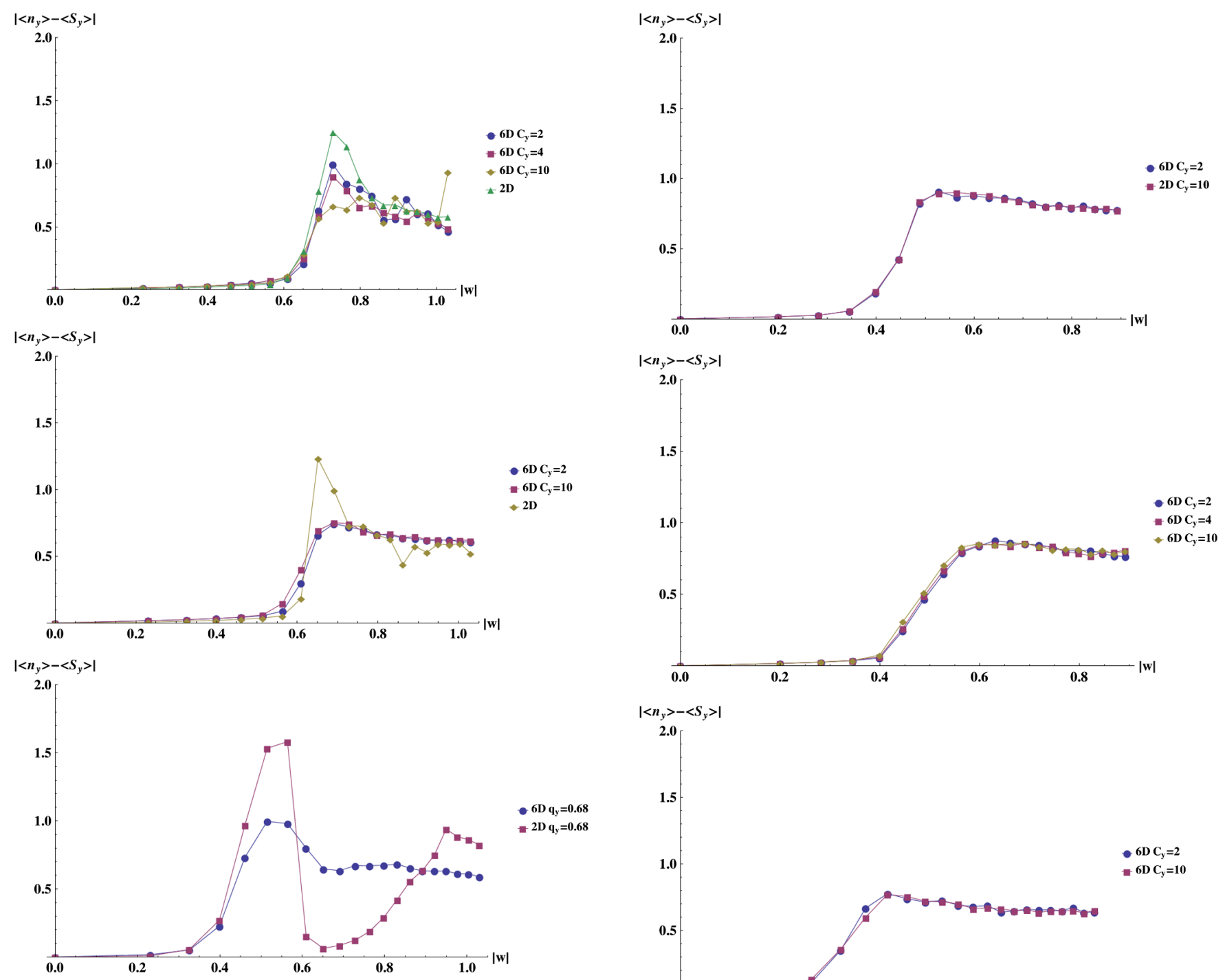

FIG. 14. Direct tracking profiles for the standard lattice crossing the $393+\nu_{y}$ resonances. We rescaled the vertical emittance to absolute resonance strength $|w|$, based on the strength of the main resonance at $10 \pi \mathrm{mm}$ mrad as calculated by DEPOL. Top: We plot four profiles with the fractional tune $\nu_{y}=0.671$, representing full $6 \mathrm{D}$ tracking with 50,400 particles at vertical chromaticities $(\mathrm{Cy}=)$ of 2,4, and 10 as well as a 2D direct tracking with zero emittance for all planes except vertical. Middle: We also plot using $\nu_{y}=0.673$ with $\mathrm{Cy}=2,10$ as well as a $2 \mathrm{D}$ direct tracking. Bottom: Here we plot using $\nu_{y}=0.680$ now only comparing $\mathrm{Cy}=2$ against 2D direct tracking. In all cases we use an acceleration rate of $\frac{d \gamma}{d t}=1.24 / \mathrm{sec}$.

effects of longitudinal motion via phase modulation of the resonances and modulation of $G \gamma$ during acceleration. However we leave this treatment for another paper and consider only 6D motion in the context of direct tracking and its relative impact on our profiles. In Fig. 14 we compare direct tracking results with nonzero emittance in only the vertical plane to full $6 \mathrm{D}$ tracking at various chromaticities for the standard lattice we used up until

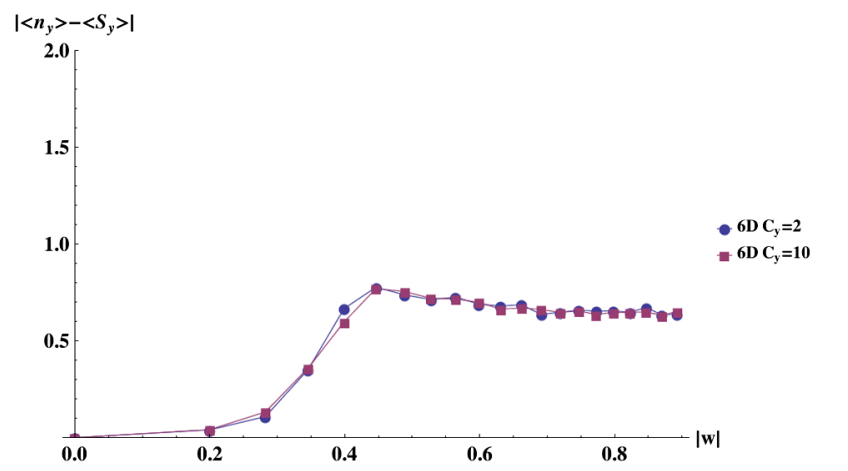

FIG. 15. Direct tracking profiles for the standard lattice crossing the $393+\nu_{y}$ resonances. We re-scaled the vertical emittance to absolute resonance strength $|w|$, based on the strength of the main resonance at $10 \pi \mathrm{mm}$ mrad as calculated by DEPOL. We plot four profiles representing full $6 \mathrm{D}$ tracking with 50,400 particles at vertical chromaticities $(\mathrm{Cy}=)$ of 2,4 , and 10 . The fractional tune is for the e-lens Yellow lattice were $\nu_{y}=0.673$ (top), $\nu_{y}=0.6705$, with $\mathrm{Cy}=2,4,10$ (middle) and for the e-lens Blue lattice $\nu_{y}=0.678$ with $\mathrm{Cy}=2,10$ (bottom). In all cases an acceleration rate of $\frac{d \gamma}{d t}=1.24 / \mathrm{sec}$ was used.

2013, (FY12 lattice). In Fig. 15 we consider 6D tracking for the Blue and Yellow e-lens lattices at different chromaticities. We observe at these tunes, there are very minor effects of larger chromaticities. The bulk effect of including longitudinal dynamics is to smooth out some of the structure of the profiles observed in 2D tracking, this also points to a possible explanation for the structure which we describe in the final section. 


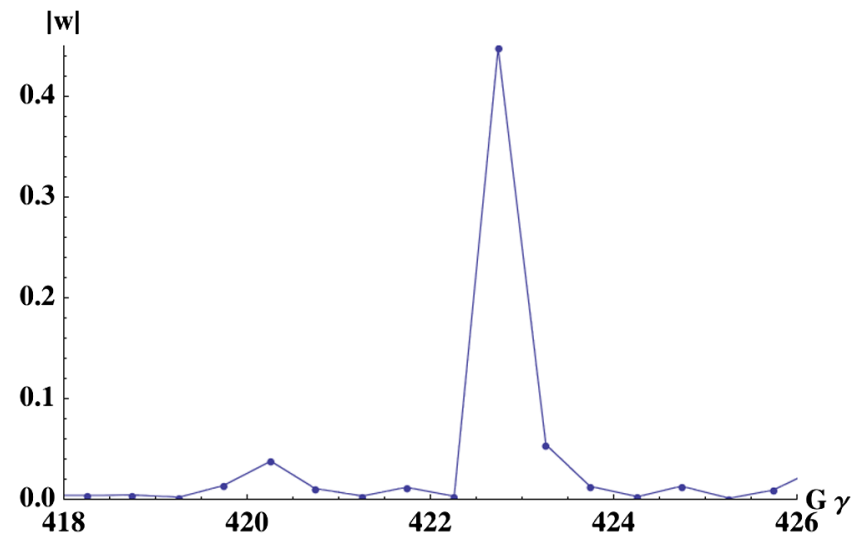

FIG. 16. Resonance structure around $393+\nu_{y}$ for the RHIC standard lattice.

\section{STUDY OF $393+\nu_{y}$ RESONANCE CROSSING FOR THE STANDARD RHIC LATTICE}

In the section on benchmarking we already considered this resonance crossing, however now we would like to study this resonance crossing in more detail since it represents one of the primary sources of polarization loss for RHIC $250 \mathrm{GeV}$ ramp and above. Revisiting the resonances structure surrounding $393+\nu_{y}$ for the standard lattice we can see (Fig. 16) that our largest neighbor appears to be the $453-\nu_{y} \approx 423.325$ resonance (which is our $K_{4}$ resonance in the previous analysis). For this resonance structure we find that we can explain most of the loss in polarization aperture by considering only two resonances, the primary $K_{1}$ and using $K_{4}$ as the secondary. This can be seen clearly in Fig. 17.

Next we scan the strength of $K_{4}\left(w_{4}\right)$ in the five resonance model (Fig. 18). We can see that if the resonance strength is reduced to $<0.01$ we essentially

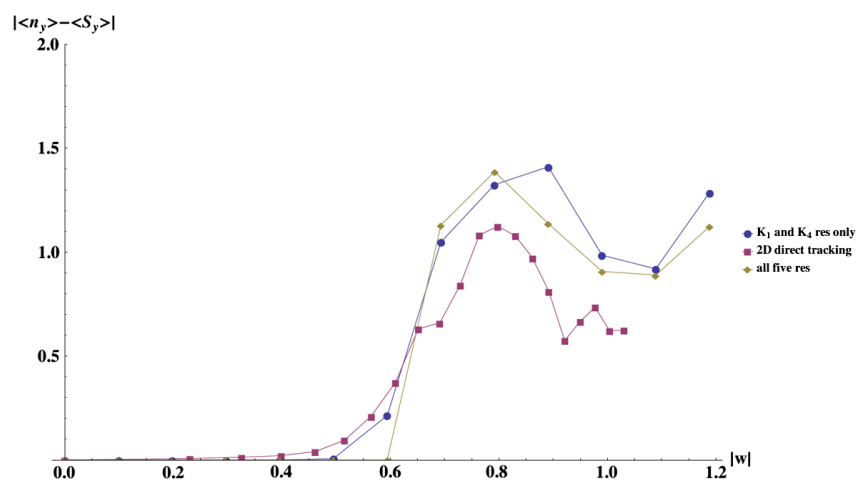

FIG. 17. Comparing a five resonance model with 2D direct lattice tracking and a $K_{1}, K_{4}$ resonance model. The simple two resonance model appears to capture most of the behavior exhibited in the five resonance and direct lattice tracking.

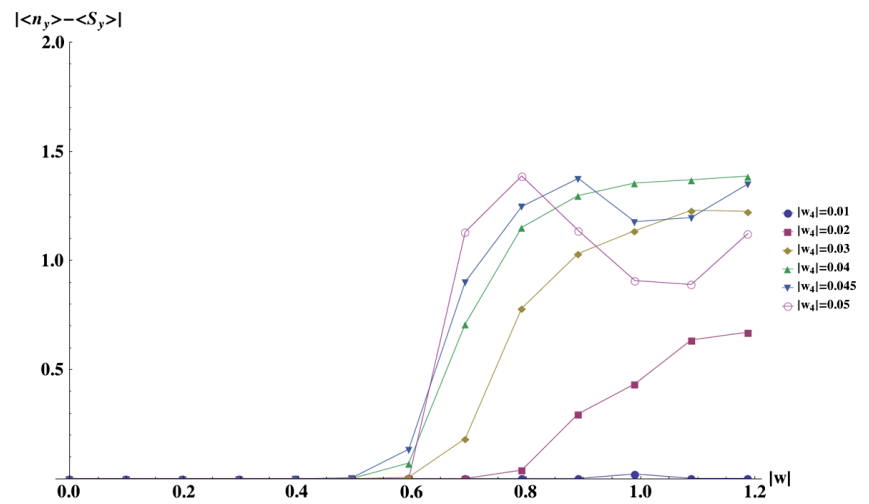

FIG. 18. The simple two resonance model $\left(K_{1}\right.$ and $\left.K_{4}\right)$. Here we scan the secondary resonance strength of $w_{4}$ for the $K_{4}$ resonance while keeping the primary resonance at $K_{1}$ fixed.

have an infinite polarization aperture (considering only intrinsic resonances). This threshold of 0.01 seems to persist even if the primary resonance increases in strength. For example in Fig. 19 we have tracked with the primary resonance $\left|w_{1}\right|=0.7$ at $10 \pi \mathrm{mmmrad}$ yet still if $\left|w_{4}\right| \leq 0.01$ the polarization aperture remains essentially infinite.
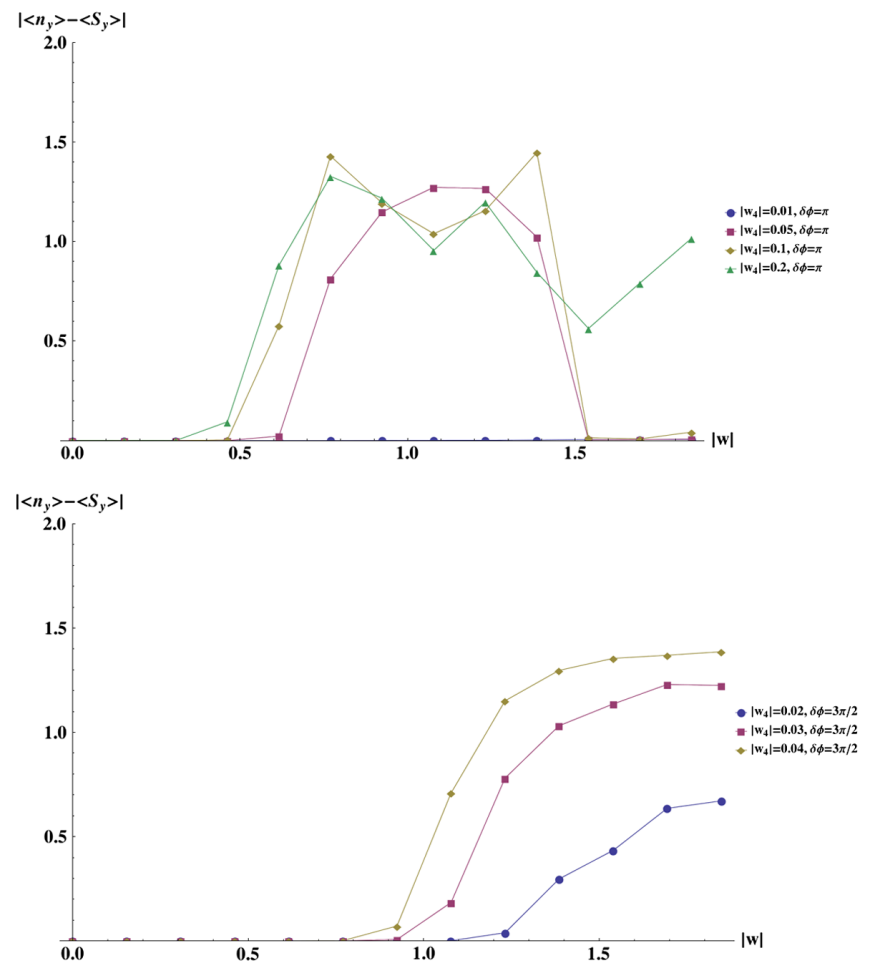

FIG. 19. Plots show polarization aperture for different $w_{4}$ values with $w_{1}=0.7$. The top are scans done with $\delta \phi=\pi$ and the bottom with $\delta \phi=3 \pi / 2$. This shows that the threshold of 0.01 for $w_{1}$ remains regardless if the primary resonance is increased. 


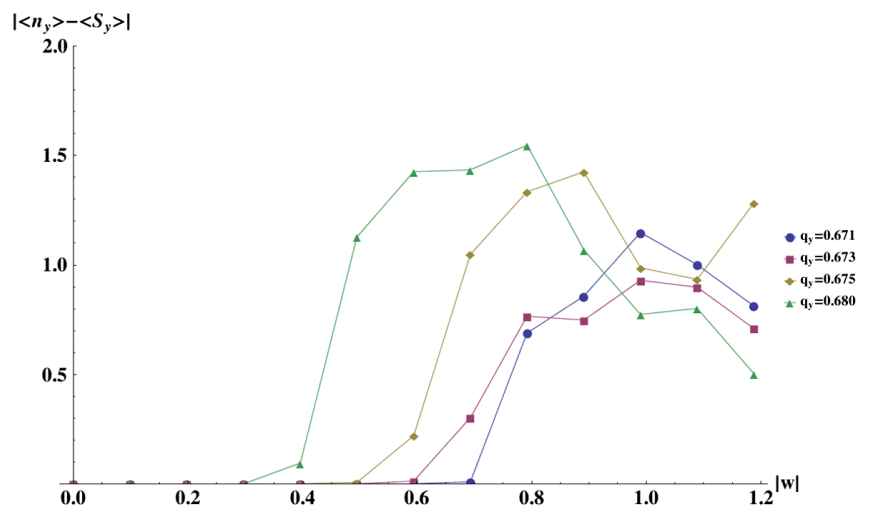

FIG. 20. Polarization response to tune when $\left|w_{4}\right|=0.04$. Shows improvement when tune approaches $2 / 3$ rds.

Finally we consider the impact of tune in the standard situation with $\left|w_{4}\right|=0.04$ in Fig. 20 and contrast that with the lattice where this $K_{4}$ resonance is reduced to 0.01 in Fig. 21. In the latter case we see what RHIC operations have now understood for several years that running nearer to the $2 / 3$ rds vertical tune offers improved polarization transmission. However in the other case when $\left|w_{4}\right|=0.01$ then polarization transmission gets worse as it approaches the $2 / 3 \mathrm{rds}$ vertical tune.

\section{A. Effect of minimizing $K_{4}$ resonance via lattice modifications}

Using a modified version of loco [18] accelerator toolbox for MATLAB we altered the standard lattice to minimize the $453-\nu_{y}$ resonance leaving all the other resonances unaffected using a spin response approach with singular value decomposition (SVD). Changing only the non focusing quadrupole (qf) and defocusing quadrupole (qd) families (whose strengths can be individually modified), we were able to reduce $\left|w_{4}\right| \approx 0.01$ with relatively minor changes to the remaining quad strengths (see Fig. 22)

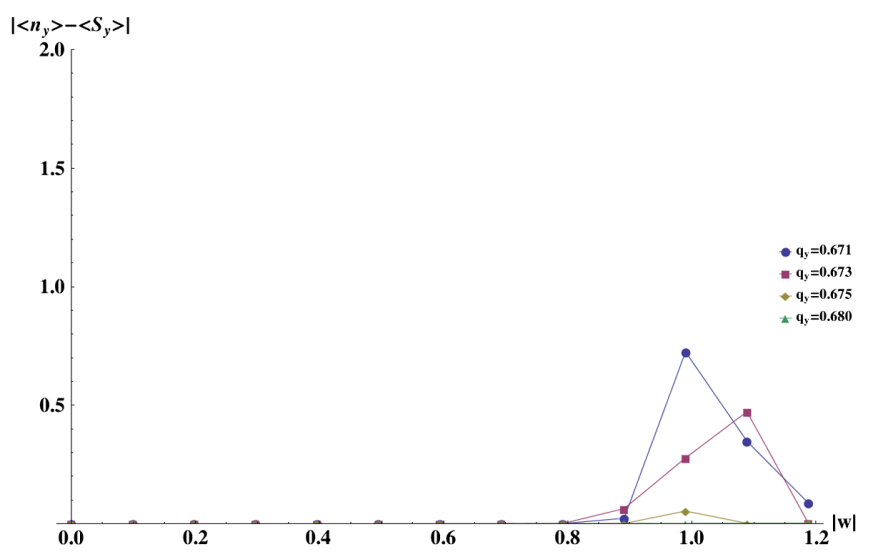

FIG. 21. Polarization response to tune when $\left|w_{4}\right|=0.01$. Shows improvement when tune moves away from $2 / 3$ rds.

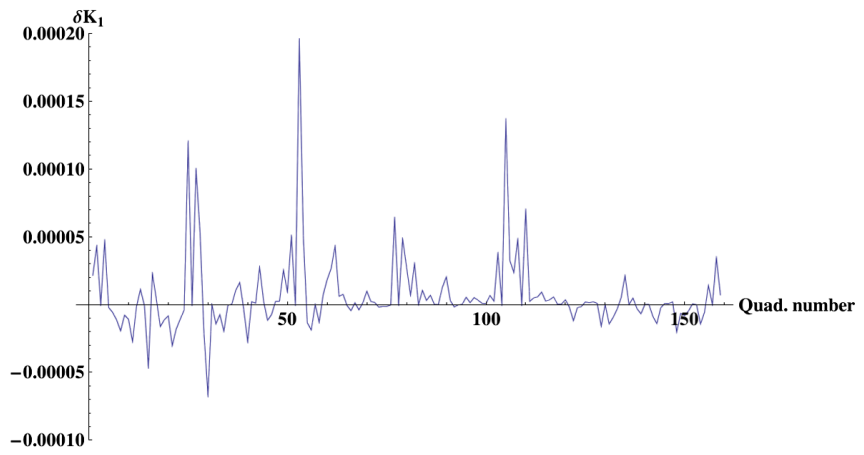

FIG. 22. Relative changes to RHIC quadrupoles excluding QF and QD tune control families required to minimize the $453-\nu_{y}$ resonance during the $393+\nu_{y}$ resonance crossing.

and modest changes to the beta functions (see Fig. 23). The effect of this change is fairly dramatic as can be seen in Fig. 24.

\section{CONJECTURE ON THE POLARIZATION APERTURE STRUCTURE}

The results of direct lattice tracking verify the structure observed in the lattice independent integration. Contrary to naive expectations polarization loss does not always rise monotonically with resonance amplitude. There can be dips and cusps in the polarization loss as a function of resonance strength. The reason for this may be rooted in the
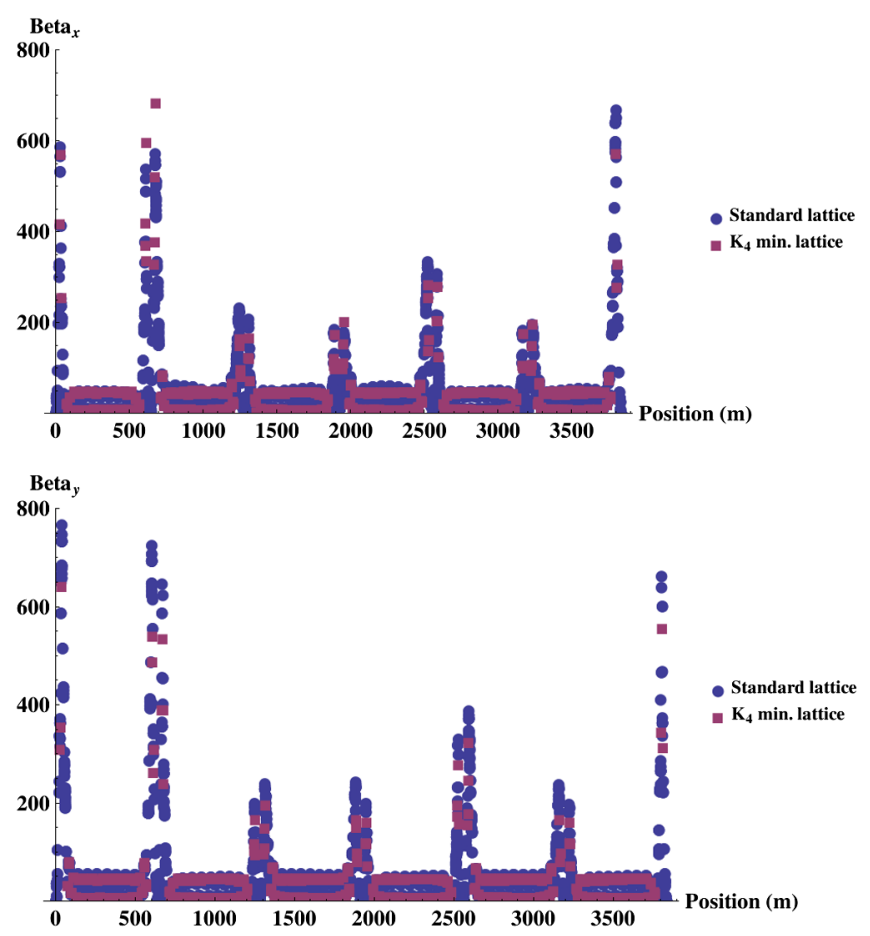

FIG. 23. Horizontal (X) Beta functions for standard lattice and $453-\nu_{y}$ minimized lattice (top), Vertical (Y) beta functions for standard lattice and $453-\nu_{y}$ minimized lattice (bottom). 


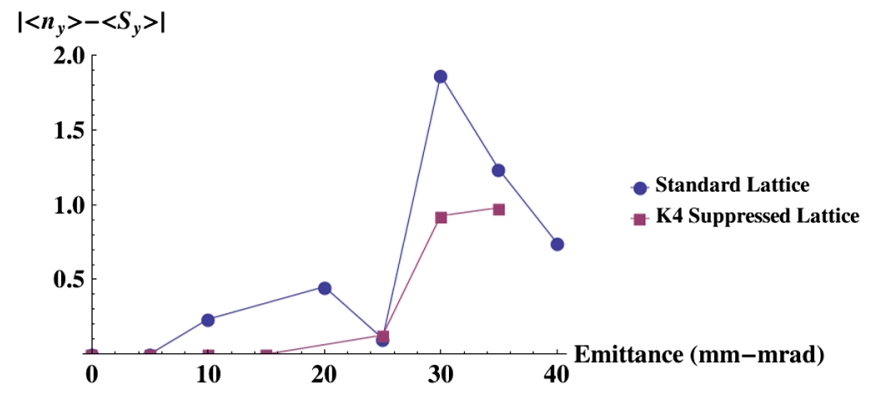

FIG. 24. Direct tracking Polarization aperture scans for standard lattice and $453-\nu_{y}$ minimized lattice.
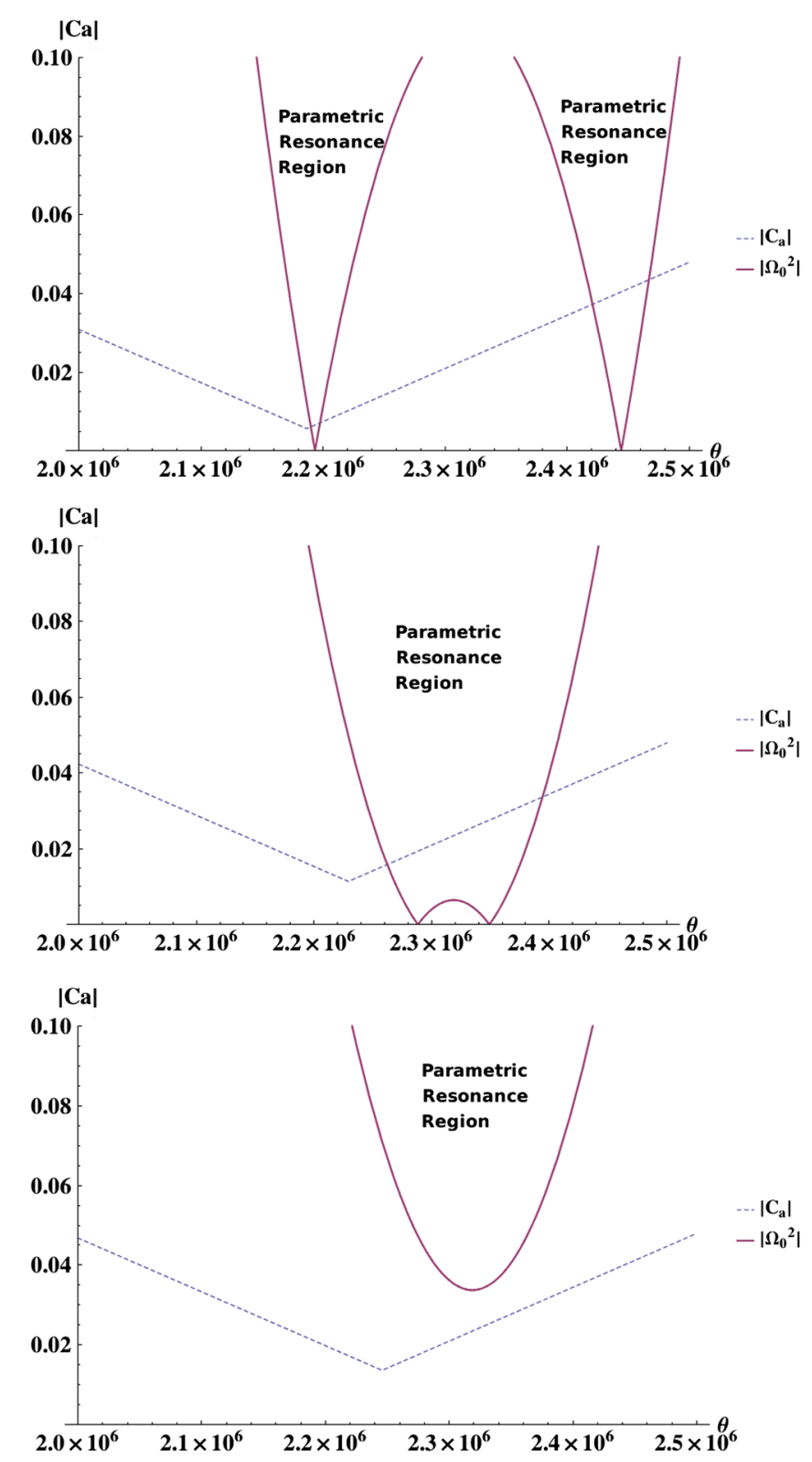

FIG. 25. These plots show the evolution of the parametric resonance area as the primary resonance strength is increased. Top: primary resonance strength 0.45 , Middle: 0.64 , Bottom: 0.7 . This type of behavior may explain the dips in polarization loss as resonance magnitude is increased. parametric resonance structure of the overlapping resonances. In a recent paper [15] it has been shown that the Thomas-BMT equation in spinor form (see Eq. (1) can be recast into a quasiperiodic Hill's like differential equation,

$$
\frac{d^{2} q}{d \theta^{2}}=\Omega^{2}(\theta) q,
$$

using the following transformations and definitions:

$$
\begin{aligned}
\beta(\theta) & =-\left(i f_{3}(\theta)+\frac{\xi^{\prime}(\theta)}{\xi(\theta)}\right) \\
\Omega^{2}(\theta) & =\frac{\beta^{\prime}(\theta)}{2}+\frac{\beta(\theta)^{2}}{4}-\frac{\xi(\theta) \xi(\theta)^{*}}{4} \\
D(\theta) & =\frac{1}{2} \int^{\theta} d \tau \beta(\tau) \\
q(\theta) & =e^{D(\theta)} \Psi_{I}^{+}(\theta) .
\end{aligned}
$$

Here the approximate kernel $\Omega^{2}(\theta)$ for two overlapping resonances is expressed as follows:

$$
\begin{aligned}
& \Omega^{2}(\theta) \approx \Omega_{0}^{2}(\theta)+C_{r}(\theta) e^{i(\delta \theta+\Delta \phi)}+C_{-r} e^{-i(\delta \theta+\Delta \phi)} \\
& \Omega_{0}^{2}(\theta)=W_{0}^{2}+C_{1} \theta+C_{2} \theta^{2} \\
& C_{a}(\theta)=\left|C_{r}(\theta)\right|+\left|C_{-r}\right| .
\end{aligned}
$$

Here $C_{a}(\theta)$ is the total amplitude for the oscillatory parts of the kernel, $\delta=K_{1}-K_{2}$ the difference between the two resonances and $\Delta \phi$ the phase difference. When considering the two resonance system without snakes, as studied in Ranjbar's paper, we see that the system behaves as a single resonance until the onset of the parametric resonance. Thus we conjecture that the mechanism for polarization loss (outside of the narrow $|w|=1$ band), with orthogonal snakes must be related to the parametric resonance. This is because we have shown that the SRM with orthogonal snakes at irrational tunes are practically resistant

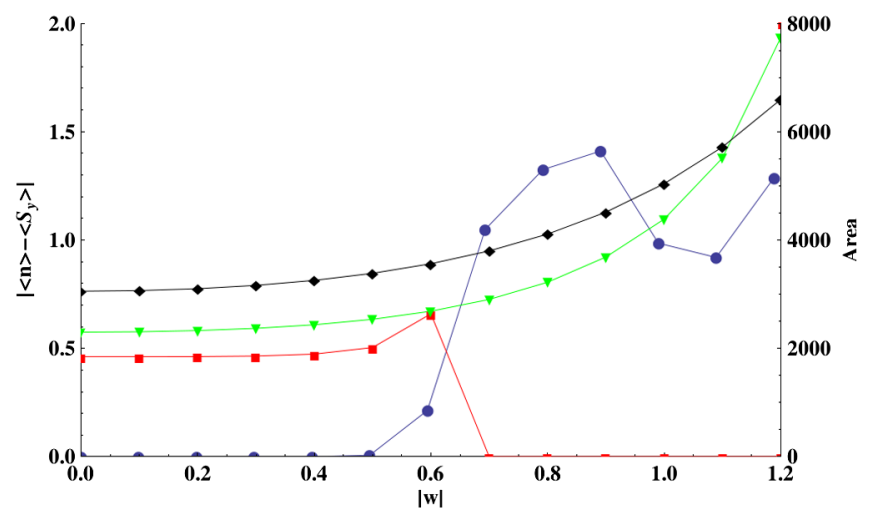

FIG. 26. Area of parametric resonance (in units of $\left|C_{a}\right| \times \theta$ ) for $1: 2$ (red square), $1: 1$ (green triangle) and $3: 2$ (black diamond) as a function of resonance strength, for $w_{1}=0.45$ and $w_{4}=0.05$ at $10 \pi \mathrm{mm}$ mrad. Comparing with polarization aperture for same resonance combination (blue circles). 


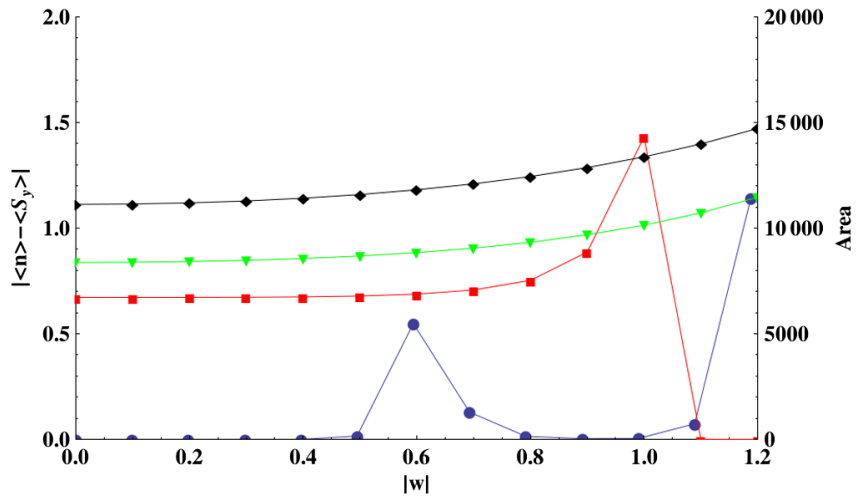

FIG. 27. Area of parametric resonance (in units of $\left|C_{a}\right| \times \theta$ ) for $1: 2$ (red square), $1: 1$ (green triangle) and $3: 2$ (black diamond) as function of resonance strength, for $w_{1}=0.45$ and $w_{0}=0.05$ at $10 \pi \mathrm{mm}$ mrad. Comparing with polarization aperture for same resonance combination (blue circles).

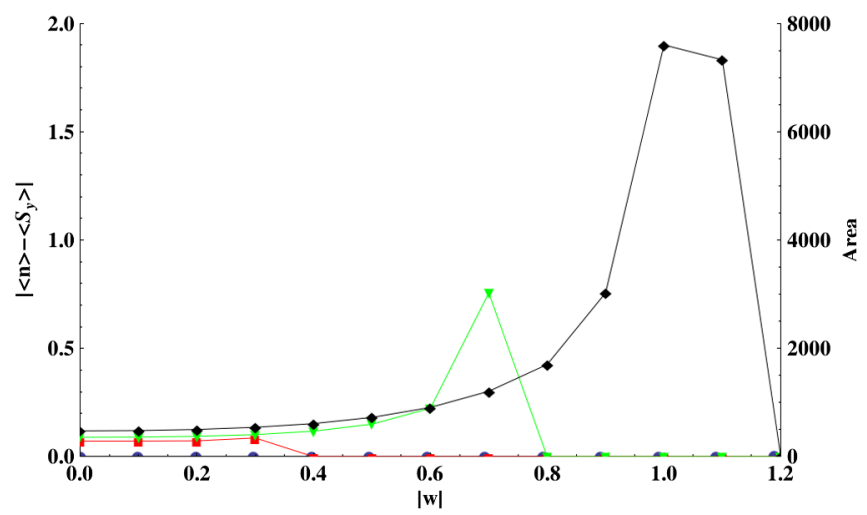

FIG. 28. Area of parametric resonance (in units of $\left|C_{a}\right| \times \theta$ ) for $1: 2$ (red square), $1: 1$ (green triangle) and $3: 2$ (black diamond) as a function of resonance strength, for $w_{1}=0.45$ and $w_{3}=0.05$ at $10 \pi \mathrm{mm}$ mrad. Comparing with polarization aperture for same resonance combination (blue circles).

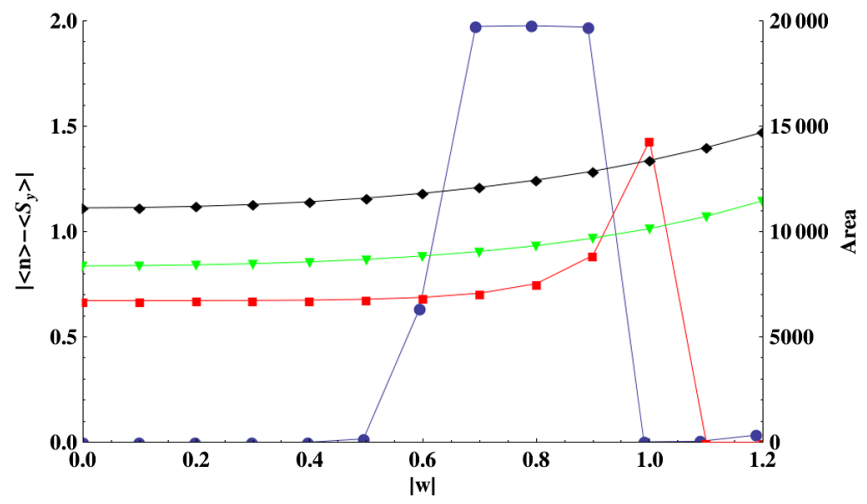

FIG. 29. Area of parametric resonance (in units of $\left|C_{a}\right| \times \theta$ ) for $1: 2$ (red square), $1: 1$ (green triangle) and $3: 2$ (black diamond) as a function of resonance strength, for $w_{1}=0.45$ and $w_{2}=0.05$ at $10 \pi \mathrm{mm}$ mrad. Comparing with polarization aperture for same resonance combination (blue circles). to depolarization and that depolarization can only occur in the presence of secondary resonance. If we refer to how in the two resonance case without snakes, the system can move in and out of a parametric resonance region as the primary resonance strength is raised while keeping the ratio of strong to weak resonance constant, we can see a possible mechanism for a similar behavior in the presences of snakes. (See Fig. 25.) In Fig. 26-29 we calculate the area of the parametric resonance enclosed between the intersection of $\left|C_{a}(\theta)\right|$ and $\left|\Omega_{0}^{2}(\theta)\right|$. We believe that this area should be proportional to the total effect of the overlapping resonance. This is motivated by the defined boundaries of the resonance tongue given by:

$$
\Omega_{0}^{2}(\theta) \approx \delta^{2} / 4 \pm\left|C_{a}\right| / 2 .
$$

Here we compare it to the structure of the polarization response for each of the neighboring resonances. We can see some qualitative similarities in their response, however the location of the peaks and valleys appear to be offset. This may be due to a systematic shift in the parametric resonance region induced by the action of the snakes.

Clearly this approach cannot explain the slight asymmetry between $K_{0}$ and $K_{2}$ resonance response since the parametric resonance areas for these two cases seem near identical. It would seem that snakes might introduce some symmetry breaking between these two resonances which is not evident in the simple two resonance model. Also this model does not include the relative phase which would be considered as a secondary effect. However it is noteworthy that the response for the $K_{3}$ resonance are small for both the tracking and parametric resonance area as well as provides a plausible physical mechanism for the dips and structure of the polarization aperture.

\section{CONCLUSION}

We have shown via direct numerical integration of the T-BMT equation for a single resonance with two orthogonal snakes at an irrational tune, that excluding narrow discontinuities at integer resonance values, the polarization transmission aperture is probably infinite, or at least well beyond any physical aperture. We have explored the critical role which interference from neighboring spin resonances can have in reducing the polarization transmission aperture during acceleration. In particular, we have found that the strength threshold for this interference appears to lie near a magnitude of 0.01 . Furthermore, we have discovered the following general principles governing the effect of neighboring resonances on a primary resonance relating to their relative location $|\Delta|$ and relative phase: $1 .|\Delta|=\left|2 q_{y}-1\right|$ can generally be ignored. 2. $|\Delta|=\left|2\left(q_{y}-1\right)\right|$ has an important role in depolarization, and its effect is independent of the resonance phase. 3. $|\Delta|=1$ has an important 
effect which depends strongly on the phase. In particular, the effects are minimized when the phase is 0 or $2 \pi$ and maximized at $\pi / 2$ and $3 \pi / 2$. 4. The effect of interfering imperfection resonances $K_{\text {imp1 }}=n+1$ and $K_{\text {imp2 }}=n-1$ are mostly independent of phase. The effect becomes significant above a magnitude of 0.01 . outside of the narrow integer band begins also at strength of 0.01 . Their relative importance is a function of their relative distance to the primary resonance and thus depends on the location of the fractional tune (closer to 0 or 1 ). 5 . The effect of an interfering coupled intrinsic spin resonance is relatively insensitive to phase. It also begins to affect transmission by a strength of about 0.01 .

Using these principles, we devised a lattice to suppress the $K_{4}$ spin resonance around the strongest spin resonance $K=393+\nu_{y}$ for the RHIC $250 \mathrm{GeV}$ acceleration ramp. This modified lattice has a greatly improved polarization transmission.

In addition, our analysis suggests that the longitudinal dynamics modifies or smooths out some of the structure of the polarization aperture, with chromaticity playing a very minor role in perturbing the polarization response.

Finally, we have also shown that the polarization transmission aperture is not always a monotonic function of emittance, and that this fact might be explained by appealing to the movement in and out of parametric resonance regions.

\section{ACKNOWLEDGMENTS}

This work is supported by BNL and the U.S. Department of Energy under Contract No. DE-SC0012704. It is also supported by the U.S. Department of Energy, Office of Science, Office of Nuclear Physics, including Grants No. DE-SC0004432 and No. DE-SC0013187. This research used resources of the National Energy Research Scientific Computing Center (NERSC), which is supported by the Office of Science of the U.S. Department of Energy under Contract No. DE-AC02-05CH11231. This research also used resources of the Oak Ridge Leadership Computing Facility at Oak Ridge National Laboratory, which is supported by the Office of Science of the U.S. Department of Energy under Contract No. DE-AC05-00OR22725.

[1] X. Gu et al., The electron lens test bench for the relativistic heavy ion collider at Brookhaven National Laboratory, Nucl. Instrum. Methods Phys. Res., Sect. A 743, 56 (2014).
[2] W. Fischer et al., arXiv:1410.5315.

[3] W. Fischer, X. Gu, Z. Altinbas, M. Costanzo, J. Hock, C. Liu, Y. Luo, A. Marusic, R. Michnoff, T. A. Miller, A. I. Pikin, V. Schoefer, P. Thieberger, and S. M. White, Operational Head-on Beam-Beam Compensation with Electron Lenses in the Relativistic Heavy Ion Collider, Phys. Rev. Lett. 115, 264801 (2015).

[4] V. H. Ranjbar et al., in Proceedings of the 4th International Particle Accelerator Conference, IPAC-2013, Shanghai, China, 2013, edited by C. Petit-Jean-Genaz et al. (JACoW, Shanghai, China, 2013), p. 1544.

[5] S. Y. Lee, Spin Dynamics and Snakes in Synchrotrons (World Scientific, Singapore, 1997).

[6] A. U. Luccio, Brookhaven National Laboratory Technical Report No. BNL-52481, 1995.

[7] A. Lehrach, A. U. Luccio, W. MacKay, and T. Roser, Particle Accelerator Conference, 2001. PAC 2001. Proceedings of the 2001 (IEEE, New York, 2001), pp. 31233125.

[8] A. U. Luccio, A. Lehrach, J. Niederer, T. Roser, M. J. Syphers, and N. Tsoupas, in Proceedings of the 1999 Particle Accelerator Conference, New York, NY, 1999, edited by A. U. Luccio and W. W. MacKay (IEEE, Piscataway, NJ, 1999), p. 1578.

[9] D. T. Abell, D. Meiser, V. H. Ranjbar, and D. P. Barber, Accurate and efficient spin integration for particle accelerators, Phys. Rev. ST Accel. Beams 18, 024001 (2015).

[10] S. R. Mane, A critical analysis of the conventional theory of spin resonances in storage rings, Nucl. Instrum. Methods Phys. Res., Sect. A 528, 677 (2004).

[11] M. Vogt, Ph.D. dissertation, Universität Hamburg, 2000.

[12] G. H. Hoffstaetter, High-Energy Polarized Proton Beams: A Modern View, Springer Tracts in Modern Physics (Springer, New York, 2006).

[13] E. D. Courant and R. D. Ruth, Brookhaven National Laboratory Technical Report No. BNL 51270, 1980.

[14] S. Blanes, F. Casas, J. A. Oteo, and J. Ros, The Magnus expansion and some of its applications, Phys. Rep. 470, 151 (2009).

[15] V. H. Ranjbar, Approximations for crossing two nearby spin resonances, Phys. Rev. ST Accel. Beams 18, 014001 (2015).

[16] V. H. Ranjbar, S.-Y. Lee, L. Ahrens, M. Bai, K. Brown, W. Glenn, H. Huang, A. U. Luccio, W. W. MacKay, V. Ptitsyn, T. Roser, and N. Tsoupas, Spin coupling resonance and suppression in the AGS, Phys. Rev. ST Accel. Beams 7, 051001 (2004).

[17] N. Khalil, Ph.D. dissertation, State University of New York, Stony Brook, NY, 2014.

[18] G. Portmann, J. Safranek, and X. Huang, Matlab based LOCO, ICFA Beam Dyn. Newslett. 44, 49 (2007). 